\title{
EDITORIAL
}

\section{IS ARTIFICIAL INTELLIGENCE REALLY A FUTURE TREND IN HEALTH CARE?}

Everyone who uses any digital platform in the daily routine has already been surprised by some sudden ad or product advertisement about which some information has been sought on the Internet. Coincidence? Of course not! This is just one example of how artificial intelligence is inserted into our daily lives. It is in the platforms for music streaming, movies, shopping for any product, in traffic applications, in the stock market. Each "like", each share, each post shows a pattern of consumer preference, a characteristic that can be used to direct advertisements in order to advertise or market a product to a specific target. This is already happening, it is not part of the future. Artificial intelligence is already part of our present.

But how do these platforms manage to "guess" our preferences or tastes and hit exactly what we were looking for? In reality nothing is guessed, it is learned. Through computer modeling, these systems learn from the examples that we ourselves give them. We feed these systems on a daily basis. Just like children, who learn many things by example (languages, for instance) before they even go to school, these systems are also capable of learning. A child learns that a dog is different from a cat when it sees examples of several dogs and several cats. So a child can learn the differences between both animals. Algorithms learn the same way, through examples. This is what we call "machine learning," a sub-area of artificial intelligence (AI). It is an advance for society, but it must be applied with ethics and transparency (see the Netflix documentary Coded Bias).
Moving away from the market sphere and thinking about health care, machine learning has also been widely employed, because these systems have the ability to learn using endless amount of patient and hospital data (Big Data). In this sense, AI-based systems have been developed aiming at improving patient care, from the organization of triage systems at clinics and hospitals, patient scheduling, organization of test result delivery, preventing errors in drug prescriptions, as well as predicting and assisting in disease diagnosis. The artificial intelligence literature in the medical field is already vast. In dentistry, research has focused on the use of convolutional neural networks (CNN) in dental radiology. Tools are produced for researchers and system developers that aim at assisting clinicians in imaging diagnosis, for example, of dental caries, periapical lesions, bone resorption, among other important outcomes.

Some companies, in Brazil and worldwide, have already seen a potential market in the application of these neural networks, and are providing software to assist in the analysis of radiographic images. Far from being able to replace health professionals, this technology should be used to improve the work of dentists and bring more security in diagnosis. Trying to replace a health professional with artificial intelligence, especially in dentistry, is impossible and not productive at all (see Eric Topol's book Deep Medicine).

Information technology as an ally will bring many benefits to dentistry, not only in radiology. The analysis of digital cohorts (electronic patient 


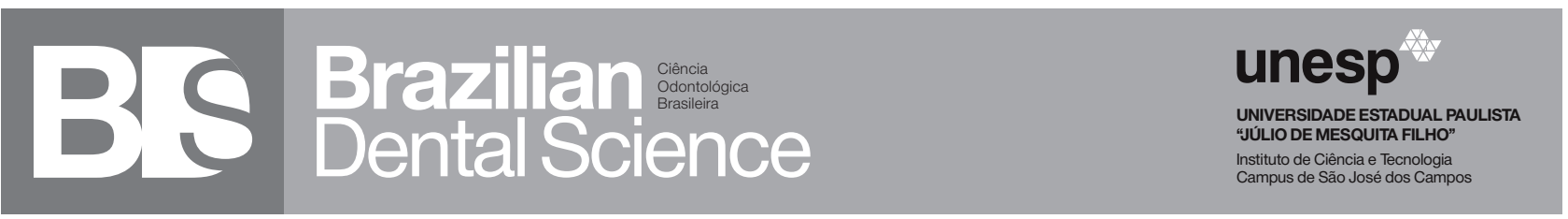

\section{EDITORIAL}

records) with machine learning algorithms can bring new insights to Science. Such algorithms are able to cross-reference thousands of predictive attributes with various endpoints to define which information is most relevant for qualitative analyses. It is the new advanced statistics.

For this reason, it is especially important to emphasize the need to build a large-scale public dental dataset to make the clinical application of AI possible. The challenge now is to improve the quality of the datasets to build really accurate machine learning algorithms. Finally, it would be very useful for dentists if these developed machine learning systems become applications that could be widely available and spread to the dental community.

The spectrum of AI is huge! Try doing a search today on some topic and wait for the algorithm to work! It will offer you all the information, based on the search example you yourself have offered! This is AI in our lives, no future, but a present!

\section{Keywords}

Artificial intelligence; Health care.

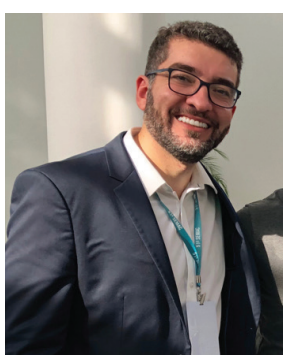

Jonas de Almeida Rodrigues

Master and PhD Degrees in Pediatric Dentistry,

São Paulo State University Júlio de Mesquita Filho, UNESP (Araraquara-SP, Brazil);

Post-Doctoral in Cariology, University of Bern, Switzerland;

Visiting Professor (CAPES PrInt), Department of Oral Diagnostics, Digital Health and Health Services Research, Charité - Berlin University of Medicine, Germany;

Associate Professor, Federal University of Rio Grande do Sul, UFRGS, Brazil.

Corresponding author: jorodrigues@ufrgs.br

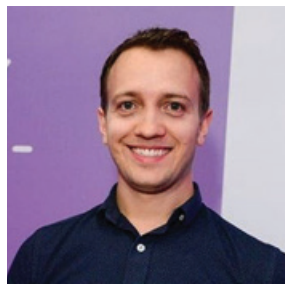

Henrique Dias Pereira dos Santos Master Degree in Computer Science, Computer Science School, Federal University of Rio Grande do Sul, UFRGS, Brazil;

PhD Degree in Computer Science, Polytechnic School, PUCRS, Brazil;

I.T. Analyst, National Center of Supercomputing. CESUP, Federal University of Rio Grande do Sul, UFRGS, Brazil

Data Scientist, Institute for Artificial Intelligence in Health, Brazil. 


\section{EDITORIAL}

\section{A INTELIGÊNCIA ARTIFICIAL É REALMENTE UMA TENDÊNCIA FUTURA NO CUIDADO EM SAÚDE?}

Todos que usam qualquer plataforma digital na sua rotina diária já foram surpreendidos com algum anúncio repentino ou propaganda de produtos sobre os quais se buscou alguma informação na internet. Coincidência? Claro que não! Esse é apenas um exemplo de como a inteligência artificial está inserida no nosso cotidiano. São as plataformas de streaming de músicas, de filmes, de compras de qualquer produto, nos aplicativos de trânsito, no mercado deações. Cada "curtida", cada compartilhamento, cada postagem mostra um padrão de gosto do consumidor, uma característica que pode ser usada para direcionar as propagandas com vistas a divulgar ou comercializar um produto com um alvo específico. Isso já acontece, não faz parte do futuro. A inteligência artificial já faz parte do nosso presente.

Mas como essas plataformas conseguem "adivinhar" os nossos gostos e acertar exatamente o que estávamos procurando? $\mathrm{Na}$ realidade nada é adivinhado, e sim aprendido. Através de modelagem computacional, esses sistemas conseguem aprender através dos exemplos que nós mesmos lhes damos. Nós alimentamos diariamente esses sistemas. Assim como as crianças, que aprendem muitas coisas através de exemplos (idiomas, por exemplo) antes mesmo de frequentarem a escola, esses sistemas também são capazes de aprender. Uma criança aprende que um cachorro é diferente de um gato quando vê exemplos de vários cachorros e vários gatos. Assim, ela consegue aprender as diferenças entre os dois animais. Os algoritmos aprendem do mesmo jeito, através de exemplos. É o que chamamos de "machine learning" ou aprendizado de máquina, uma subárea da inteligência artificial (IA). É um avanço para a sociedade, mas que deve ser aplicado com ética e transparência (vide o documentário Coded Bias da Netflix).

Saindo um pouco da esfera do mercado e pensando em cuidado em saúde, o aprendizado de máquina também tem sido bastante empregado, isso porque esses sistemas têm a capacidade de aprender utilizando uma infindável quantidade de dados dos pacientes e dos hospitais (Big Data). Neste sentido, sistemas baseados em IA têm sido desenvolvidos com o objetivo de aprimorar o atendimento aos pacientes, desde a organização dos sistemas de triagem de clínicas e hospitais, de agendamento de pacientes, de organização de envio de resultados de exames, prevenir erros na prescrição de medicamento, além de predizer e auxiliar no diagnóstico de doenças. A literatura de inteligência artificial na área médica já é vasta. Na Odontologia, as pesquisas têm focado no uso de redes neurais convolucionais (CNN) na radiologia odontológica. São produzidas ferramentas para pesquisadores e desenvolvedores de sistemas que visam auxiliar o clínico no diagnóstico por imagem, por exemplo, da cárie dentária, de lesões periapicais, reabsorções ósseas, dentre outros desfechos importantes.

Algumas empresas, do Brasil e do mundo, já viram na aplicação dessas redes neurais um mercado em potencial e estão fornecendo softwares para auxiliar na análise das imagens radiográficas. Longe de conseguir substituir os profissionais, essa tecnologia deve ser utilizada 


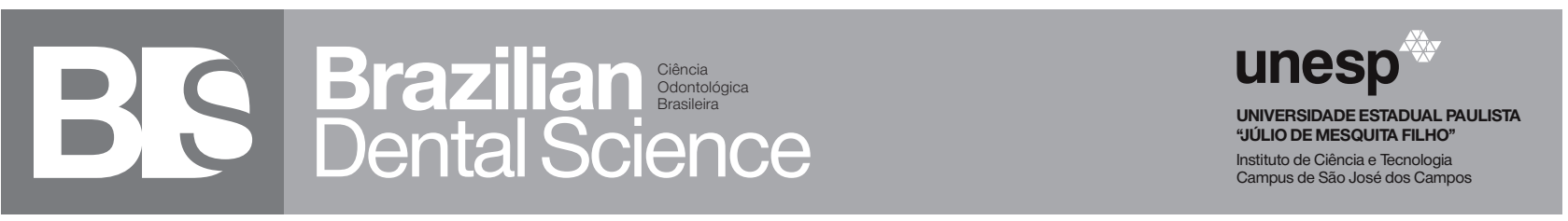

\section{EDITORIAL}

para aprimorar o trabalho dos dentistas e trazer mais segurança no diagnóstico. Tentar substituir um profissional da saúde com inteligência artificial, especialmente na odontologia, é impossível e nada produtivo (vide o livro Deep Medicine de Eric Topol).

A tecnologia da informação como uma aliada irá trazer muitos benefícios para a odontologia, não só na radiologia. A análise das coortes digitais (prontuários eletrônicos de pacientes) com algoritmos de machine learning podem trazer novos insights para a ciência. Tais algoritmos são capazes de cruzar milhares de atributos preditivos com vários desfechos para definir quais informações são mais relevantes para as análises qualitativas. É a nova estatística avançada.

Por esta razão, é especialmente importante enfatizar a necessidade de construir um conjunto de dados públicos odontológicos em larga escala para tornar possível a aplicação clínica da IA

Em suma, o desafio agora é melhorar a qualidade dos conjuntos de dados para construir algoritmos de aprendizagem de máquinas realmente precisos. Finalmente, seria muito útil para os dentistas se estes sistemas desenvolvidos de aprendizagem de máquinas se tornassem aplicações que poderiam ser amplamente disponíveis e difundidas para a comunidade odontológica.

O espectro da IA é enorme! Experimente fazer uma busca hoje sobre o tema e aguarde o algoritmo trabalhar! Ele vai te oferecer todas as informações, baseando-se no exemplo de busca que você mesmo ofereceu! Essa é a IA nas nossas vidas, nada de futuro, e sim um presente!

\section{Palavras-chave}

Inteligência artificial; Cuidado em Saúde.

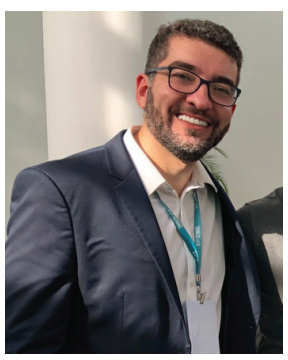

Jonas de Almeida Rodrigues

Mestre e Doutor em Odontopediatria pela Faculdade de Odontologia da UNESP (Araraquara-SP, Brasil);

Pós-Doutorado em Cariologia na Universidade de Berna, Suíça;

Professor Visitante (CAPES PrInt) no Departamento de Diagnóstico Oral, Saúde Digital e

Pesquisa em Serviços de Saúde, na Universidade Pesquisa em Serviços de Saúde, na
de Medicina de Berlim, Alemanha;

Professor Associado na Universidade Federal do Rio Grande do Sul, UFRGS, Brasil.

Autor correspondente: jorodrigues@ufrgs.br

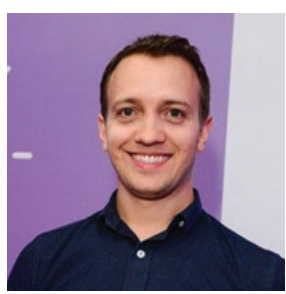

Henrique Dias Pereira dos Santos Mestre em Ciência da Computação pela Faculdade de Informática da Universidade Federal do Rio Grande do Sul, UFRGS, Brasil;

Doutor em Ciência da Computação pela Escola Politécnica da Pontifícia Universidade Católica do Rio Grande do Sul, PUCRS, Brasil;

Analista de T.I., Centro Nacional de Supercomputação, CESUP, Universidade Federal do Rio Grande do Sul, UFRGS, Brasil;

Cientista de Dados no Instituto de Inteligência Artificial na Saúde, Brasil. 